Vietnam Journal of Mechanics, NCST of Vietnam Vol. 24, 2002, No 3 (133-141)

\title{
EXTENSION OF METHOD OF MOMENT EQUATION TO NONLINEAR DETERMINISTIC VIBRATIONS
}

\author{
NGuyen Dong Anh ${ }^{1}$ and Ninh Quang $\mathrm{HAI}^{2}$ \\ 1 Institute of Mechanics, Hanoi Vietnam \\ ${ }^{2}$ Hanoi Architectural University, Vietnam
}

\begin{abstract}
The paper present the so-called "an extended averaged equation approach" to the investigation of nonlinear vibration problems. The numerical results in analysing the vibration systems with weak, middle and strong non-linearity show the advantages of the method.

Keywords. Extended averaged equation, moment equation (ME) method, nonlinear vibration
\end{abstract}

\section{Introduction}

In recent decades, a great number of achievements in nonlinear oscillation field has been obtained. There are several documents specializing in approximate techniques for solving deterministic and / or random vibration problems, for instance, see [1-6] and see [7-9], respectively. Many outstanding analysis methods as well as numerical methods are established. As results, significant characteristics of nonlinear systems subject to deterministic or random excitations are discovered. These properties have been effectively applied to many engineering fields, such as problems of vibration for machines, traffic means, civil-engineering etc...

However, there still exist many problems that need to be investigated, namely, there is a number of gaps between weakly nonlinear systems and strongly nonlinear ones, between deterministic systems and stochastic ones. Some well-known methods which can be applied to stochastic systems (for instant, the F-P-K equation method, the Gaussian closure method...) normally cannot be used for deterministic ones and vice verse (for example, the harmonic balance method,...) due to the fact that many principal concepts in stochastic vibrations do not make sense in deterministic fields. Furthermore, it is obviously that some outstanding methods such as: the method of small parameter, the averaging method, the Gaussian equivalent linearization method, the Gaussian closure method... are very effective in analyzing weakly nonlinear systems. Nevertheless, the accuracy of these techniques decreases rapidly when non-linearity of the systems increases.

Several well-known analysis methods are very useful in theoretical investigations but can hardly be combined with computers (for instant, the averaging method...). In contrast, many numerical methods are very effective in calculating and making simulations but they have a limited ability to find out common properties or rules 
governing systems due to its weak point in analysis. Therefore. "semi numericalsemi analysis methods" need introducing by researches.

In addition, vibration problems of engineering systems with strong non-linearity especially the ones under earthquakes, storms... have been of great concern. Unfortunately, most of well-known approximate methods such as method of small parameter, averaging method [1-6] for investigating non-linear vibration phenomena are only applicable to weakly nonlinear systems.

Method of moment equation (ME) is an effective tool analysis of random vibration problems. It can give good approximate solution for strong nonlinear systems if an adequate choice of closure can be made [10-12].

The aim of the paper is to extend the method of $\mathrm{ME}$ to the field of deterministic vibrations. First, an extended averaged equation for deterministic systems is presented and then, for illustration, some free oscillations of the Duffing system are investigated. Finally, the advantages of the proposed method are shown in the numerical results.

\section{Extend averaged equation for deterministic system}

Consider a nonlinear system

$$
\dot{Z}=f(Z), \quad Z=\left(z_{1}, z_{2}, \ldots, z_{n}\right)
$$

where $f(Z)=\left(f_{1}(Z), f_{2}(Z), \ldots, f_{n}(Z)\right)$ is a nonlinear function vector of the vector $Z$. Together with the original system $(2.1)$, we introduce its corresponding linear system

$$
\dot{X}=A X, \quad X=\left(x_{1}, x_{2}, \ldots, x_{n}\right), \quad A=\left\{a_{i j}\right\} \quad(i, j=1,2, \ldots, n),
$$

here $A$ is an unknown constant matrix, which will be determined from equations proposed later by the method. For an arbitrary differentiable function $\Psi(Z, X)$ one gets

$$
\frac{d \Psi}{d t}=\sum_{i=1}^{n}\left[\frac{\partial \Psi}{\partial z_{i}} f_{i}(Z)+\frac{\partial \Psi}{\partial x_{i}}\left(\sum_{j=1}^{n} a_{i j} x_{j}\right)\right] .
$$

Denote the averaged operator (Borgoliubov \& Mitropolskii [1-3])

$$
\langle.\rangle=\lim _{T \rightarrow+\infty} \frac{1}{T} \int_{0}^{T}(.) d t .
$$

Suppose that

$$
\left\langle\frac{d \Psi}{d t}\right\rangle=0 .
$$


So, one gets

$$
\sum_{i=1}^{n}\left\langle\frac{\partial \Psi}{\partial z_{i}} f_{i}(Z)\right\rangle+\sum_{i=1}^{n}\left\langle\frac{\partial \Psi}{\partial x_{i}}\left(\sum_{j=1}^{n} a_{i j} x_{j}\right)\right\rangle=0 .
$$

It is supposed that all the averaged terms in the Eq. (2.6) exist. In the paper, we consider $\Psi=z_{k}^{m} z_{s}^{n}, \Psi=z_{k}^{m} x_{s}^{p}, \Psi=x_{k}^{m} x_{s}^{p}$, here $k, s, m, p=0,1,2, \ldots$ It should be remarked that, the equation (2.6) - (a set of equations) could be referred to as extended averaged equation, which is similar to the moment equation in the theory of random vibrations, where the averaged operator is taken in the probabilistic meaning. The first advantage of the Eq. (2.6) is that this equation is exact one if the condition (2.5) is satisfied. The second advantage is that the Eq. (2.6) can be applicable to weak and strong nonlinear systems since the condition of small nonlinearity of the system is not used for establishing the Eq. (2.6). Furthermore, the Eq. (2.6) contains both the response $Z(t)$ of the original system and the response $X(t)$ of its corresponding linear system. Thus, it can express the link between the responses. Consequently, it allows us to makes choices of the variable $Z(t)$ in some optimal ways. On the other hand, one might suppose that the accuracy of the technique would be better if more averaged equations could be satisfied. In order to satisfy more equations, the original variable $Z(t)$ is proposed to be taken in form of a nonlinear vector function of variable $X(t)$

$$
Z=\Phi(X, \alpha),
$$

here $\boldsymbol{\alpha}$ also is an unknown vector. Now, $Z(t)$ is not a solution of a linear system any longer, so it can express nonlinear properties of the original system, which is used to be ignored when applying some classical methods. In this paper, we confine ourselves to establishing the response of the nonlinear Eq. (2.1) in a polynomial form. Consequently, the matrix $A$ and the vector $\alpha$ of the polynomial are unknown, which will be determined by solving a certain closed set of equations formed from the Eqs. (2.6) (and initial conditions of the Eq. (2.1), if there are).

It seems that the averaged equations (2.6) may give good approximate solutions for both weak and strong nonlinear systems. These advantages will be illustrated by some following examples.

\section{Duffing oscillator}

Consider a free oscillator of the Duffing system governed by the following equation

$$
\left\{\begin{array}{llll}
\ddot{z}+\beta z+\varepsilon z^{3}=0 & & & \text { (a) } \\
& \text { with } & z(0)=z_{0} & \text { (b) } \\
& \dot{z}(0)=0 & \text { (c) }
\end{array}\right.
$$


Introduce its corresponding linear system

$$
\ddot{x}+k^{2} x=0 \text {. }
$$

In this cases, the Eq. (2.6) takes the form

$$
\left\langle\frac{\partial \Psi}{\partial z} \dot{z}\right\rangle-\left\langle\frac{\partial \Psi}{\partial \dot{z}} f(z)\right\rangle+\left\langle\frac{\partial \Psi}{\partial x} \dot{x}\right\rangle-\left\langle\frac{\partial \Psi}{\partial \dot{x}} k^{2} x\right\rangle=0
$$

where

$$
f(z)=\beta z+z^{3}
$$

Taking the "lowest" polynomial functions $\Psi(z, \dot{z}, x, \dot{x})$ from (3.3), one gets following equations, for example,

$$
\begin{aligned}
& \text { for } \Psi=z \dot{z} \quad\left\langle\dot{z}^{2}\right\rangle-\left\langle z\left(\beta z+\varepsilon z^{3}\right)\right\rangle=0 \\
& \text { for } \Psi=x \dot{z} \quad\left\langle x\left(\beta z+\varepsilon z^{3}\right)\right\rangle-\langle\dot{z} \dot{x}\rangle=0
\end{aligned}
$$

The equation (3.5) is conventional averaged one for original variable $z, \dot{z}$ while the equation (3.6) etc... contain $z, \dot{z}, x, \dot{x}$. They show links between the variables. In order to close a set of averaged equations one needs some additional relationships between the variables. For instance, in the averaging method one puts

$$
z(t)=x(t)
$$

here $x(t)$ - the solution of the linear system (3.2), takes the form

$$
x=a \cos \varphi ; \quad \varphi=k t .
$$

It should be noted that, according to $(3.7), z(t)$ can be considered as a polynomial with only one term of $x(t)$ (the first-degree one) and the coefficient of the polynomial is equal to unit. It follows from (3.1b), (3.7) and (3.8) that $a=z_{0}$. Then, there is only one unknown $k$ (or the period $T$ ) left. Thus, only one equation from the hierarchy of the averaged equations is needed. One can use the Eq. (3.5), which now coincides with Eq. (3.6)

$$
\left\langle\dot{x}^{2}\right\rangle-\left\langle x\left(\beta x+\varepsilon x^{3}\right)\right\rangle=0 .
$$

For a $T$-period solution $z(t)$, one has

$$
\langle.\rangle=\frac{1}{T} \int_{0}^{T}(.) d t=\frac{1}{2 \pi} \int_{0}^{2 \pi}(.) d \varphi .
$$

Therefore, it follows from (3.8)-(3.9)

$$
\frac{k^{2} a^{2}}{2}-\frac{\beta a^{2}}{2}-\frac{3 \varepsilon a^{4}}{8}=0
$$


then

$$
k^{2}=\beta+\frac{3 \varepsilon a^{2}}{4} .
$$

Denoting $A=\varepsilon z_{0}^{2}$, one gets the period of the one-term-solution

$$
T_{i}=\frac{2 \pi}{k}=\frac{2 \pi}{\sqrt{\beta+\frac{3 A}{4}}} .
$$

It should be remarked that for the Eq. (3.1) one has the exact period $T_{E}([1,4])$ for $\beta=1$

$$
T_{E}=\frac{4}{\sqrt{1+A}} \int_{0}^{\pi / 2} \frac{d \theta}{\sqrt{1-m \sin ^{2} \theta}}, \quad \text { where } \quad m=\frac{A}{2(1+A)} .
$$

Now, the response of the nonlinear Eq. (3.1) is proposed to be a cubic polynomial

$$
z(t)=x(t)+\alpha x^{3}(t)
$$

Thus, there are 3 unknowns $a, \alpha$ and $k$ (or $T$ ). They can be found from a closed set of 3 equation (3.1b), (3.5)-(3.6). The Eq. (3.1b) gives

$$
\alpha=\frac{\left(z_{0}-a\right)}{a^{3}} .
$$

Substituting (3.16) into (3.5) and (3.6), we obtain following equations for $a$ and $T$

$$
\begin{aligned}
& 1280 \pi^{2} a^{2}-64 \beta a^{2} T^{2}-7 \varepsilon a^{4} T^{2}-1536 \pi^{2} z_{0} a-128 \beta z_{0} a T^{2}-20 \varepsilon z_{0} a^{3} T^{2} \\
& -320 \beta z_{0}^{2} T^{2}-42 \varepsilon z_{0}^{2} a^{2} T^{2}-84 \varepsilon z_{0}^{3} a T^{2}-231 \varepsilon z_{0}^{4} T^{2}+2304 \pi^{2} z_{0}^{2}=0, \\
& 128 \pi^{2}-32 \beta T^{2}-3 \varepsilon a^{2} T^{2}-21 \varepsilon z_{0}^{2} T^{2}=0 .
\end{aligned}
$$

\begin{tabular}{|c|c|c|c|c|c|}
\hline \multirow[b]{2}{*}{$\varepsilon$} & & & & \multicolumn{2}{|r|}{ able 1} \\
\hline & $T_{E}$ & $T_{p}$ & error of $T_{p}$ & $T_{1}$ & error of $T_{1}$ \\
\hline 0 & 6.28319 & 6.28319 & & 6.28319 & \\
\hline 0.01 & 6.25976 & 6.25976 & $-7.10^{-8 \%} \%$ & 6.25976 & $-0.0001 \%$ \\
\hline 0.1 & 6.06066 & 6.06065 & $-0.00006 \%$ & 6.06004 & $-0.01 \%$ \\
\hline 1 & 4.76802 & 4.76734 & $-0.014 \%$ & 4.74964 & $-0.39 \%$ \\
\hline 4 & 3.17972 & 3.17724 & $-0.078 \%$ & 3.14159 & $-1.2 \%$ \\
\hline 10 & 2.19183 & 2.18897 & $-0.13 \%$ & 2.15511 & $-1.68 \%$ \\
\hline 40 & 1.15186 & 1.14984 & $-0.17 \%$ & 1.12849 & $-2.03 \%$ \\
\hline 100 & 0.73628 & 0.73490 & $-0.18 \%$ & 0.720731 & $-2.11 \%$ \\
\hline 1000 & 0.23435 & 0.23389 & $-0.19 \%$ & 0.229277 & $-2.17 \%$ \\
\hline
\end{tabular}

Finally, the solution $z(t)$ of the original nonlinear system (3.1) can be obtained from (3.15). The period $T_{1}$, the period $T_{p}$ obtained by the Eq.s (3.17)-(3.18) are compared with the exact ones are shown in the Table 1 for $\beta=1$ with different values of $\varepsilon$. 
The cases $\beta=1$

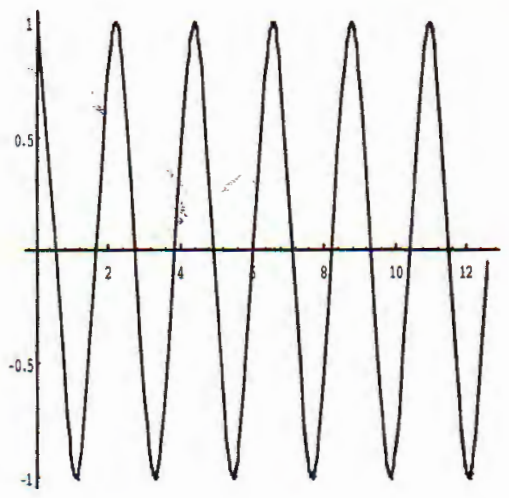

Fig. $1 a \quad \varepsilon=10$

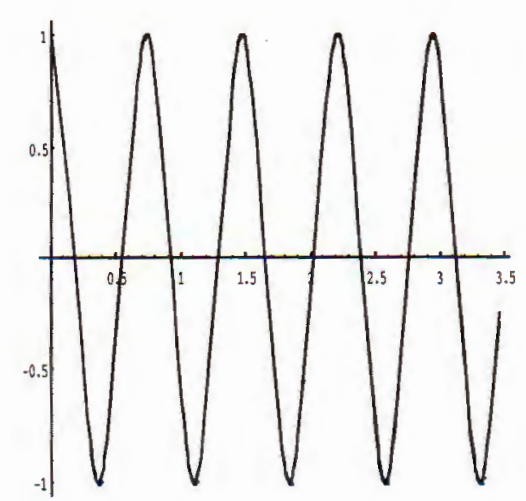

Fig. $1 c \varepsilon=100$

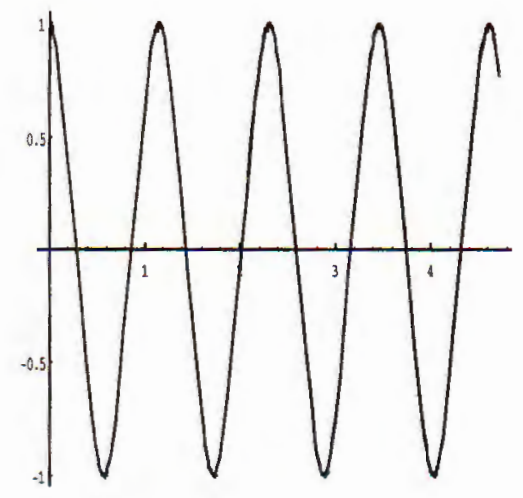

Fig. $1 b \quad \varepsilon=40$

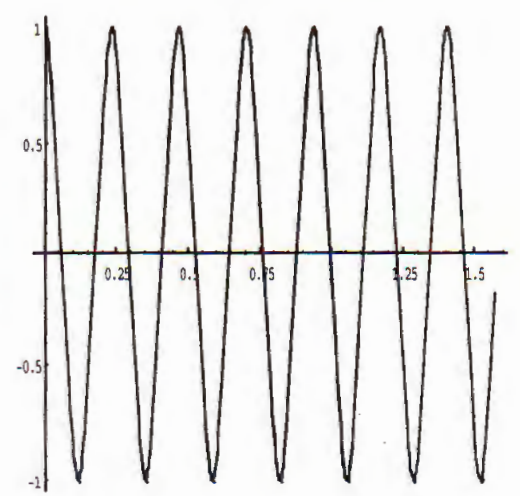

Fig. $1 d \quad \varepsilon=1000$

The cases $\beta=-1$

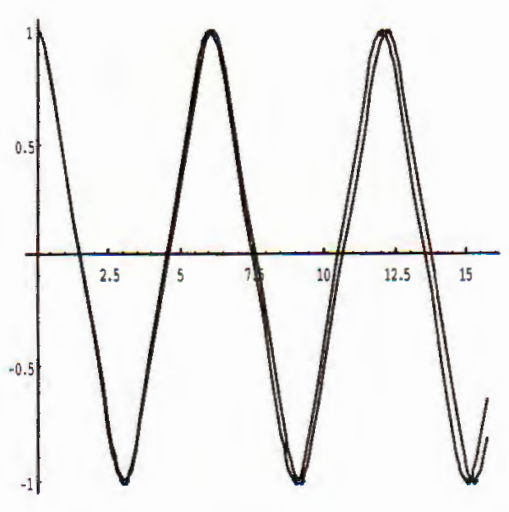

Fig. $2 a \quad \varepsilon=3$

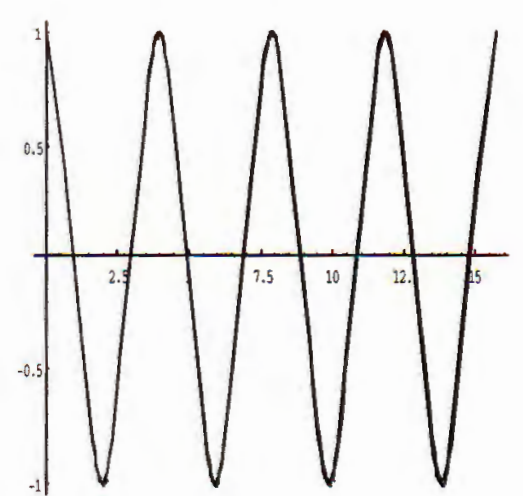

Fig. $2 b \quad \varepsilon=5$ 


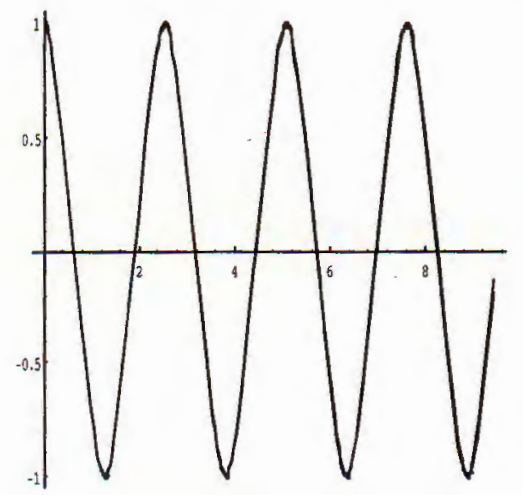

Fig. $2 c \varepsilon=10$

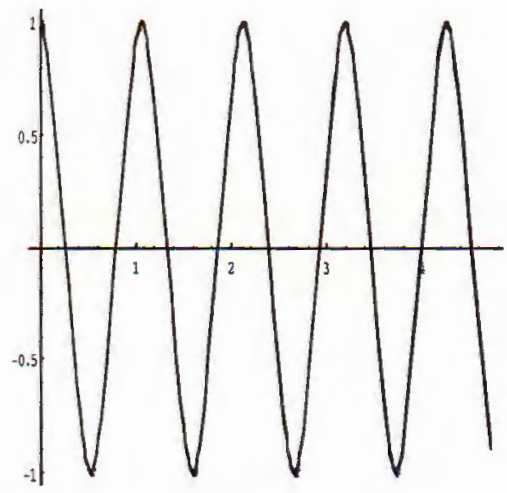

Fig. $2 d \varepsilon=100$

The cases $\beta=0$

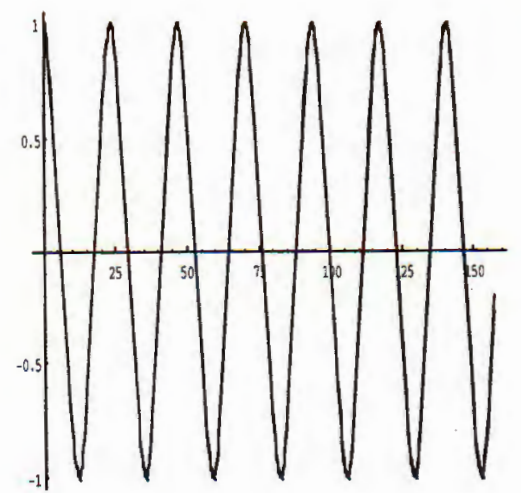

Fig. $3 a \quad \varepsilon=0.1$

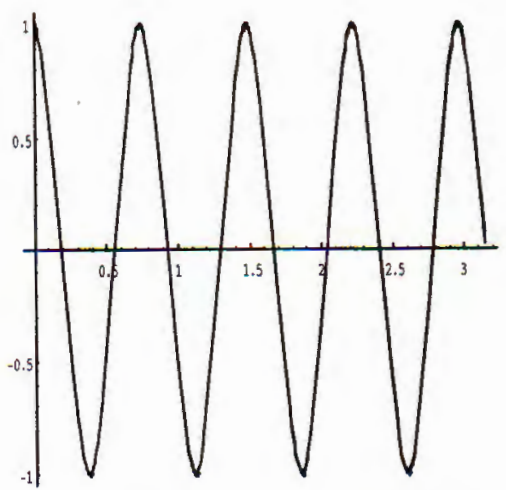

Fig. $3 c \quad \varepsilon=100$

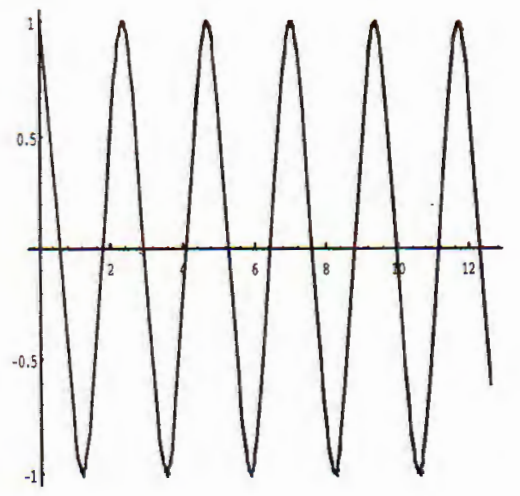

Fig. $3 b \quad \varepsilon=10$

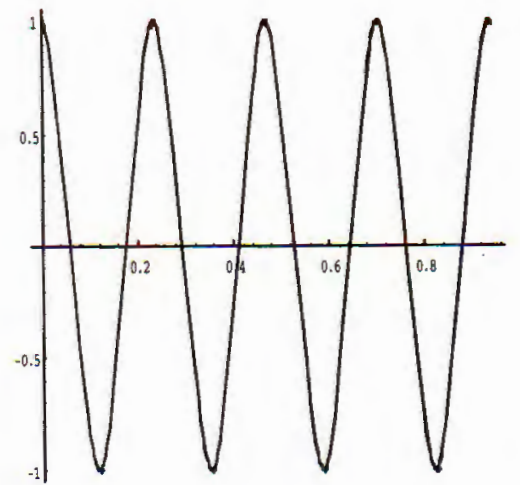

Fig. $3 d \quad \varepsilon=1000$ 
It can be seen from the Table 1 that the periods $T_{p}$ are very close to $T_{E}$. For further illustration, pairs of the graphs obtained by the proposed method and by numerical simulation are shown in the following figures for the cases: $\beta=1, \beta=-1$ and $\beta=0$ with different values of $\varepsilon$.

It can be seen from the Figs. 1, 2, 3 (a. b, c, d) that each pair of the graphs obtained by the proposed method and by the numerical simulation approximately coincide with each other in the case of weak non-linearity and in that of strong non-linearity.

\section{Conclusion}

In the paper, the original and its corresponding linear equations are considered simultaneously. As the result, the so-called extended averaged equation is established. It is similar to the moment equations in the theory of random vibrations, where the averaged operator is taken in the probabilistic meaning. In addition, the solution of the nonlinear system is proposed to be a polynomial of the solution of its corresponding linear system. Based on this equation, the correlation between the original variable $Z(t)$ and the variable $X(t)$ is shown. Thus, a possible way to determine the polynomial coefficients and the linear system can be derived. In addition, the technique is quite simple since it can use properties of the harmonic functions, although the calculations are more complicated than the averaging method. The proposed method has been effectively applied to the free oscillations of the Duffing system. However, the technique should be tested for other non-linear systems and some related questions may aside: Which set of extended averaged equations and which form of polynomials should be chosen to get a better approximate solution?

The research has been supported by a grant of the Fundamental Research Program in Natural Sciences.

\section{REFERENCES}

1. Bogoliubov N. N., Mitropolskii Yu. A. Asymptotic methods in the theory of nonlinear oscillations, $4^{\text {th }}$ ed., Moscow, 1974, (in Russian).

2. Mitropolskii Yu. A., Nguyen Van Dao, Nguyen Dong Anh. Nonlinear oscillations in the systems of arbitrary order, Kiev, 1992, (in Russian).

3. Mitropolskii Yu. A., Nguyen Van Dao. Applied asymptotic methods in nonlinear oscillations, Kiev-Hanoi, 1994.

4. Nayfeh A. H. Perturbation Methods. John \& Sons 1973.

5. Nayfeh A. H. and Mook D. T. Nonlinear Mechanics, Wiley, 1979.

6. Volosov V. M. and Morgunov V. I. The Method of Averaging in the Theory of Non Linear Systems Izd-vo, MGU, Moscow, 1971. 
7. Anh N. D., Schiehlen W. An approach to the problem of closure in the non-linear stochastic mechanics. Int. J. of Meccanica, 29 (1994), 109-123.

8. Grigoriu M. Applied Non-Gaussian Processes, PTR Prentice Hall, Englewood Cliffs, NJ, (1995).

9. Wojtkiewicz S. F., Spencer Jr. B. R., Bergman L. A. New insights on the application of moment closure methods to non-linear stochastic systems. In Proc. of IUTAM Symposium on Non-Linear Stochastic Dynamics, Eds. Naess A. and S., Krenk, Kluwer (1976), pp. 479-488.

10. N. D. Anh, N. Q. Hai. A Technique of closure using a polynomial function of Gaussian Process. Journal of Probabilistic Engineering Mechanics, Elsevier Science Ltd. 15 (2000), 191-197.

11. Nguyen Dong Anh \& Ninh Quang Hai. A technique for solving non-linear systems subject to random excitations of mechanical systems, in Proc of IUTAM Symposium, NOMES, Eds N. V. Dao and Kreuzser, 2000 Kluwer Academic Publishers, pp. 217-226.

12. N. D. Anh, N. Q. Hai. Two techniques of closure in random vibration problems, Proc. of the $2^{\text {nd }}$ Vietnam-Japan Symposium on Advances in Applied Electromagnetics and Mechanics, January 19-21, 2000 (accepted for publication).

Received July 15, 2001

MỞ RộNG PHUƠNG PHÁP PHƯONG TRÌNH MÔMEN CHO HỆ DAO ĐộNG PHI TUYẾN TIỀN ĐỊNH

Bài báo đề xuất phương pháp "phương trình trung bình suy rộng" - một sự mở rộng phương pháp phương trình mô men của dao động ngẫu nhiên. Đồng thời phương pháp cũng đề nghị mô tả nghiệm của hệ phi tuyến bằng một hàm phi tuyến của nghiệm của hệ tuyến tính tương ứng. Phương pháp được trình bày tổng quát về phương diện lý thuyết. Để minh họa, đã áp dụng cho các dao động tự do của hệ Duffing có thành phần phi tuyến bậc ba với thành phần lực đàn hồi tuyển tính dương, âm và bằng không. Các kết quả số cho thấy ưu điểm của phương pháp đối với các hệ có độ phi tuyến yếu, vừa và mạnh. 